\title{
Regulation and manipulation of angiogenesis in the primate corpus luteum
}

\author{
Hamish M. Fraser and Stephen F. Lunn \\ MRC Human Reproductive Sciences Unit, Centre for Reproductive Biology, \\ 37 Chalmers Street, Edinburgh EH3 9ET, UK
}

\begin{abstract}
Intense physiological angiogenesis occurs during the early stages of luteal development, providing a model in which the complex processes regulating the angiogenic pathway may be studied. Here, a working hypothesis is presented to explain the diverse changes in the vasculature of the corpus luteum that occur over a short period, based around changes in vascular endothelial growth factor, the angiopoietins and matrix metalloproteinases. An illustration is given of how angiogenesis can be monitored in a primate model and how the role of individual angiogenic factors such as vascular endothelial growth factor may be explored in vivo. Because of the marked effect of inhibition of angiogenesis on luteal function, it is predicted that the normal processes of follicular development, ovulation and luteal function could all be profoundly influenced by the manipulation of angiogenesis.
\end{abstract}

Recent advances in the localization of angiogenic factors in the ovary, and determination of their temporal relationships with ovarian function, permits the construction of a working model for the regulation of normal, physiological angiogenesis (Hanahan, 1997; Maisonpierre et al., 1997; Duncan, 2000; Wiegand et al., 2000). As there are many potential regulators of angiogenesis, it is essential to establish appropriate in vivo models to determine whether a specific factor, or its receptor(s), has a major or modulatory role, or is of no physiological significance. This review discusses a current working model and its use in the exploration of the regulation of ovarian angiogenesis in vivo. A non-human primate, the marmoset (Callithrix jacchus), has been used in this work, which focuses on angiogenesis in the corpus luteum. The use of primate models allows the identification of the prospects for manipulation of angiogenic factors in humans, and thus forms a bridge between basic research and clinical application. The regulation of luteal function differs markedly between primates and other mammals; in addition, the luteal phase of the laboratory rodent is of much shorter duration. The reader is referred to the reviews of Abulafia and Sherer (2000), Augustin (2000), Redmer and Reynolds (1996) and Reynolds et al. (2000) for further information on ovarian angiogenesis.

Three techniques have been used to monitor the process of angiogenesis both throughout the normal ovarian cycle and after the manipulation of angiogenic growth factors. First, incorporation of administered bromodeoxyuridine (BrdU) into deoxyribonucleic acid (DNA) has been used as an indicator for proliferating cells. Second, identification and quantification of endothelial cells has been undertaken

Email: h.fraser@hrsu.mrc.ac.uk using the specific endothelial cell markers CD31 or factor VIII. Third, changes in luteal function resulting from treatment have been assessed by measurement of plasma progesterone concentrations.

\section{Monitoring changes in ovarian angiogenesis}

Angiogenesis in the corpus luteum has its origins in the vasculature of the developing follicle (Suzuki et al., 1998). Dual labelling of BrdU-positive proliferating cells with CD31 for the endothelial cells demonstrates that primordial and primary follicles do not have their own blood supply. However, as the follicle develops, and a morphologically distinct thecal compartment is formed, endothelial cells are recruited from the pre-existing vasculature, which is abundant in the adjacent ovarian stroma (Fig. 1a). Proliferation in both the thecal and granulosal compartments gradually increases to a maximum in the late preantralearly antral stage of development, but blood vessels lying within the thecal layer are denied entry into the avascular granulosa cell layer by the presence of the basement membrane, which separates the two compartments (Fig. 1a). This arrangement is maintained through the antral stages of development (Fig. 1b). After ovulation and release of the ovum and antral fluid, the follicle collapses inwards and the thecal layer, together with its attendant blood vessels, is situated within the folds of the granulosal compartment. Loss of integrity of the basement membrane occurs, accompanied by extensive tissue re-modelling, with the commencement of invasion of the differentiating granulosa lutein cell-containing region by new blood vessel development from the pre-existing thecal vasculature (Fig. 1c). The next few days are associated with a period of intensive angiogenesis, and the newly formed vasculature 

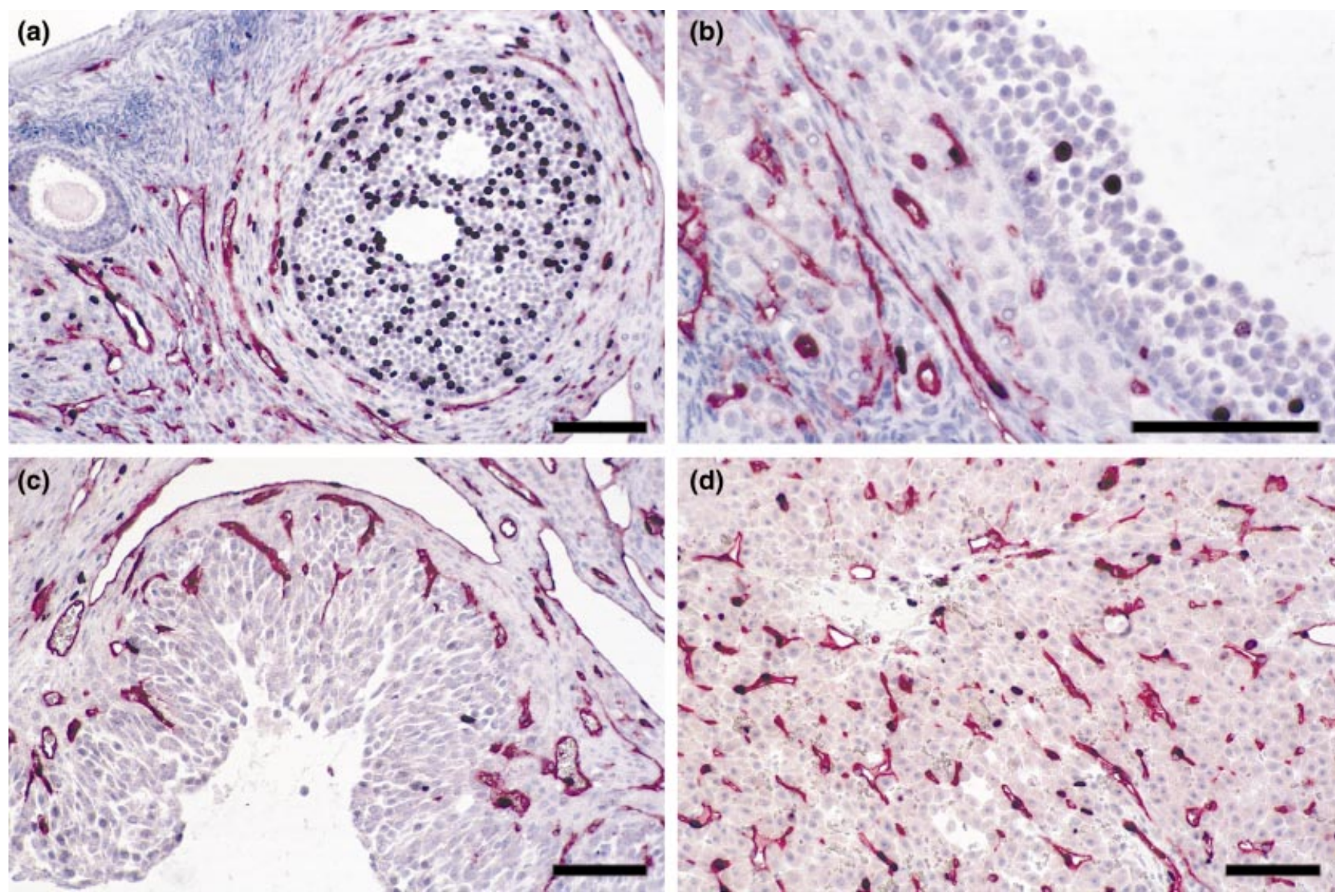

Fig. 1. Dual labelling of bromodeoxyuridine (BrdU)-positive proliferating cells (blue-black nuclei) with CD31 for the endothelial cells (red) in the marmoset (Callithrix jacchus) ovary. (a) Section of ovary showing a primary follicle without a blood supply surrounded by abundant stromal vasculature. In the neighbouring early antral follicle, the granulosa cell layer is undergoing rapid proliferation and the thecal compartment contains numerous blood vessels. (b) Pre-ovulatory follicle showing how the vasculature remains restricted to the thecal layer. (c) Recently ovulated follicle showing how the thecal layer has collapsed inwards and, together with its attendant blood vessels, is situated within the folds of the granulosal compartment. (d) A few days later, after a period of intensive angiogenesis, the vasculature has spread throughout the corpus luteum. Scale bars represent $100 \mu \mathrm{m}$.

extends throughout the tissue (Fig. 1d). By the midluteal phase, all of the fully differentiated lutein cells are in contact with the endothelium and the mature corpus luteum has the highest blood flow of any tissue in the body.

Immunofluorescence can be used to detect both BrdUand CD31-labelled cells, and also allows the percentage of proliferating cells that are endothelial to be calculated. At all stages of the luteal phase, $>80 \%$ of the dividing cells in the corpus luteum are endothelial cells (Dickson and Fraser, 2000). Establishment of the proliferation index through the early, mid- and late luteal phases shows that it is in the early luteal phase that the intense angiogenesis is taking place (Dickson and Fraser, 2000) (Fig. 2). Although nonpathological angiogenesis also occurs in the endometrium and placenta and during wound healing, such intense angiogenesis is unique to the corpus luteum in the adult
(Eberhard et al., 2000), and occurs in all mammals studied to date, including women (Rodger et al., 1997).

Endothelial cells, once formed, normally remain functional for 2-3 years but in the corpus luteum these newly formed, immature blood vessels regress within a matter of weeks during structural luteolysis in the non-conceptual cycle (Gaytán et al., 1999). However, the function of the corpus luteum is to produce the progesterone required for establishment and maintenance of early pregnancy and, in the presence of the conceptus from the fertile cycle, the life span of the corpus luteum, with its attendant vasculature, is further prolonged. Therefore, within a relatively short period, the corpus luteum must accommodate a period of a few days of intense angiogenesis, controlled regression of the vasculature in the non-fertile cycle during luteolysis, or stabilization and maturation of the vasculature during luteal rescue in the conceptual cycle. 


\section{Regulation of luteal angiogenesis}

Changes in potential regulators of luteal angiogenesis strongly indicate that it is regulated by $\mathrm{LH}$, particularly since most of the major potential factors are produced by the lutein cells, which respond to changing LH stimulation (Fig. 3 ). In most other tissues, hypoxia is the major trigger for the synthesis of angiogenic factors, and its potential role in luteal angiogenesis has been reviewed by Reynolds et al. (2000).

The origin of some of the principal factors involved in the regulation of angiogenesis in the corpus luteum, and how changes in their activity and interaction may explain the divergent processes of blood vessel formation, stabilization and degeneration are illustrated (Fig. 3). According to this model, lutein cells must stimulate angiogenesis by causing sprouting from the proximal blood vessels to obtain the oxygen, nutrients and hormone precursors necessary to synthesize large amounts of progesterone. Under the influence of angiogenic growth factors, the matrix metalloproteinases (MMPs) are activated such that the extracellular matrix (ECM) associated with the blood vessels is degraded at specific regions. This process is held in check by the production of tissue inhibitors of MMPs (TIMPs) (Duncan, 2000). For example, TIMP-1 is produced in massive amounts by the lutein cells throughout the luteal phase. Vascular endothelial growth factor (VEGF, also known as vascular permeability factor, VPF) is undoubtedly the major specific stimulator of endothelial cell proliferation, acting through two tyrosine kinase receptors, VEGF-R1 (fms-like tyrosine kinase, Flt-1) and VEGF-R2 (kinase insert domain-containing region, KDR) (Otani et al., 1999). However, blood vessel formation also involves other essential factors to co-ordinate the process, including the angiopoietins Ang-1 and Ang-2, which are of particular interest with respect to regulation of the luteal vasculature because there is evidence that they function to influence the stabilization of blood vessels on the one hand, and their destabilization on the other (Maisonpierre et al., 1997; Wiegand et al., 2000). Ang-2, acting through its tyrosine kinase receptor Tie-2, aids the action of VEGF by reducing endothelial contact with the ECM, and hence with adjacent endothelial cell interactions. In contrast, Ang-1, acting via the same receptor, acts to enhance the stability of the newly formed blood vessels. The expression patterns for the mRNAs for these regulatory factors also show considerable variation, adding to the complexities of these local interactions. However, since divergent processes are likely to occur at any particular time in the same tissue this is perhaps not surprising. Thus, while TIMP-1 is expressed overall in both the theca- and granulosa-derived luteal cells in women, the expression of Ang-2 is punctate, being observed in only a minority of both lutein and endothelial cells (Wulff et al., 2000).

The functions of the luteal endothelial cells are also influenced by a number of regulators that are likely to have a solely paracrine role. For example, there is evidence that

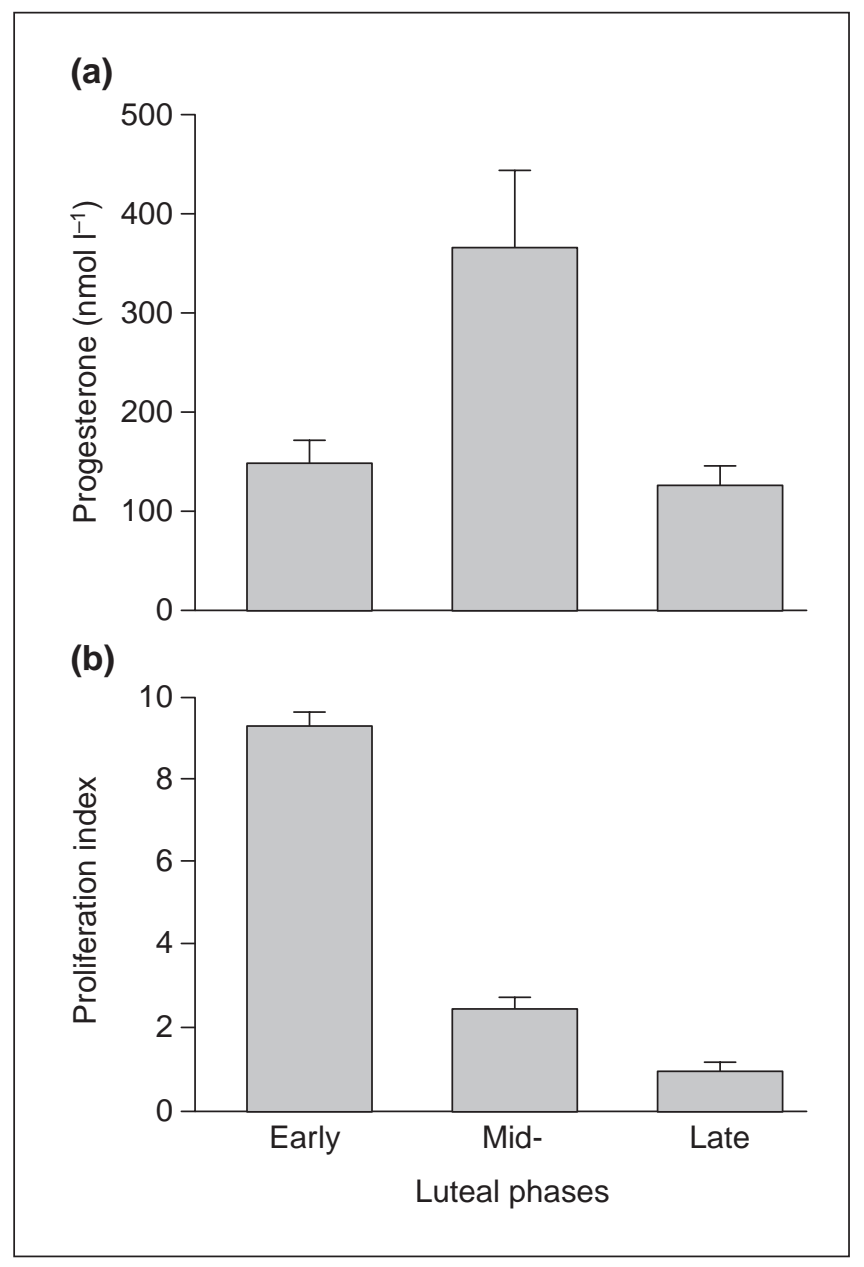

Fig. 2. Changes in plasma progesterone concentrations and cell proliferation calculated from bromodeoxyuridine (BrdU) incorporation into luteal cells during the early, mid- and late luteal phases of marmosets. (Adapted from Dickson and Fraser, 2000, with permission.)

insulin-like growth factors (IGFs) and their binding proteins (IGFBPs) may be important effectors of luteal function. IGFBP-3 mRNA is expressed in the endothelium of the marmoset (Fraser et al., 1998) and in human corpus luteum (Fraser et al., 2000a). The signal is strong during the early luteal phase, but decreases during the mid- and late luteal phases.

During luteal rescue, after conception, it appears that there is no associated burst of intense angiogenesis (Christenson and Stouffer, 1996a; Rodger et al., 1997). This finding may indicate that the vascular tree required for the corpus luteum of pregnancy is already established by this time, and that it is the stabilization rather than the extension of the vasculature that is required at this stage. The production of those factors associated with maintenance of the vasculature may be continued under the influence of human chorionic gonadotrophin (hCG), with Ang-1 acting to stabilize the vessels, possibly by recruiting pericytes (Fig. 3). 


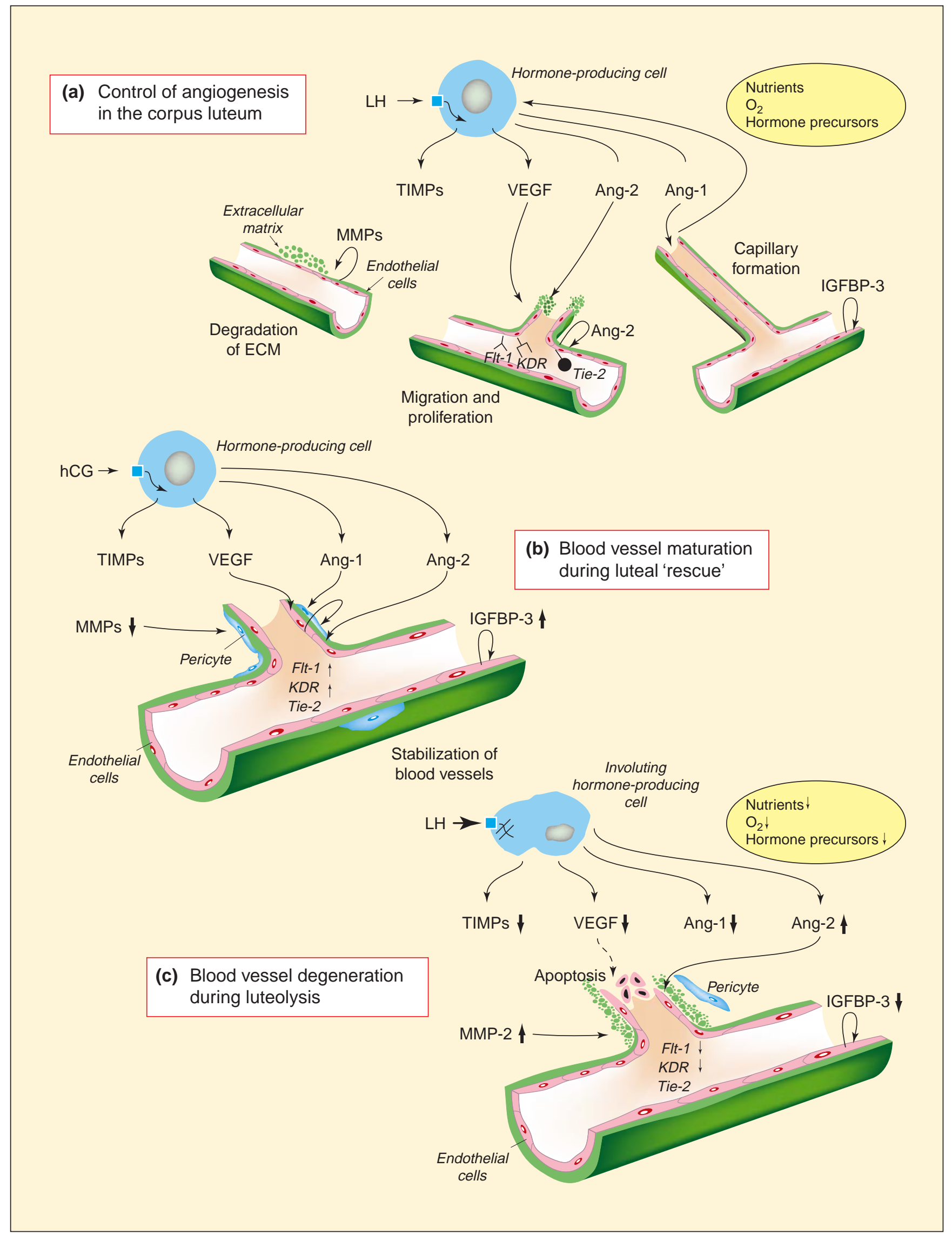


The need for degradation of the ECM is diminished, and at least one of the MMPs (MMP-2) decreases in the hCGrescued corpus luteum (Duncan, 2000). Administration of hCG caused a marked increase in the amounts of IGFBP-3 mRNA in luteal endothelial cells, comparable to that observed during the early luteal phase (Fraser et al., 2000a). These observations raise the possibility that the regulated expression of endothelial IGFBP-3 plays a role in controlling angiogenesis and cell responses in the human corpus luteum by autocrine-paracrine mechanisms.

In the non-fertile cycle, the responsiveness of the lutein cells to LH decreases at the end of the luteal phase, as does their synthetic activity for growth and survival factors, such as VEGF (Fig. 3), and the lutein cells involute (Maisonpierre et al., 1997; Fraser et al., 1999b). There is little evidence that luteal vascular degeneration is a trigger for luteolysis (Young et al., 2000), but the onset of the luteal vascular degeneration would undoubtedly lead to a reduction in the supply of oxygen and nutrients, and perhaps contribute to lutein cell death. At least one MMP has been shown to increase in the late luteal phase, and this probably contributes to an irreversible breakdown of the ECM with its attendant loss of cell-cell contact (Duncan, 2000). If luteolysis is associated with a decrease in both Ang-1 and VEGF expression co-incident with an increase in Ang-2, as has been shown in rats (Maisonpierre et al., 1997), then Ang-2 should further destabilize the blood vessels, leading to loss of pericyte contact (Goede et al., 1998), endothelial cell detachment and death by apoptosis (Wiegand et al., 2000). Such endothelial apoptotic death is readily observed in guinea-pigs (Azmi and O'Shea, 1984), although it is rarely observed in the bovine (Modlich et al., 1996) or monkey corpus luteum (Fraser et al., 1999b). Whatever the form of endothelial cell death, the result is regression of the luteal blood vessels.

\section{Manipulation of angiogenesis}

Some of the interactions proposed have been investigated in both rats (Ferrara et al., 1998) and marmosets (Dickson and Fraser, 2000; Fraser et al., 2000b) by direct manipulation in vivo. In marmosets, the intense angiogenesis in the early corpus luteum has been selected as a target and the effect of suppressing endogenous LH by GnRH antagonist treatment starting on day 1 after ovulation investigated (Dickson and

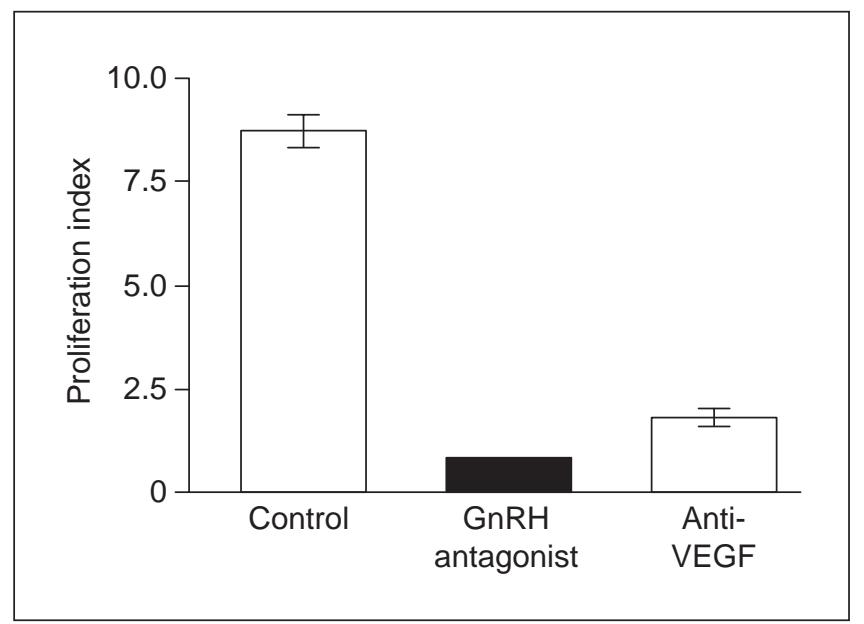

Fig. 4. Changes in proliferation index in the marmoset corpus luteum of the early luteal phase after treatment with either $\mathrm{GnRH}$ antagonist or monoclonal antibodies to vascular endothelial growth factor (VEGF). (Adapted from Dickson and Fraser, 2000; and Fraser et al., 2000b.)

Fraser, 2000). Examination of corpora lutea $48 \mathrm{~h}$ later revealed a $90 \%$ reduction in the number of BrdU-positive cells, showing that the occurrence of early luteal angiogenesis is dependent on LH stimulation of the lutein cells (Fig. 4). It is likely that this withdrawal of LH interferes with the production of angiogenic factors. For example, in vitro studies indicate that there is a temporal relationship between LH, VEGF and endothelial cell proliferation (Christenson and Stouffer, 1996b; Hazzard et al., 1999). However, withdrawal of LH in vivo also has the potential to disrupt cell-cell interactions likely to be necessary for angiogenesis; morphological assessment of the lutein cells showed that their integrity had been lost in GnRH-treated marmosets (Dickson and Fraser, 2000).

The exciting research undertaken with respect to development of angiogenic inhibitors, principally for use in the treatment of solid tumours, must be used to identify the importance of specific angiogenic regulators. Such materials can be categorized into: (i) compounds developed for other indications, but subsequently found to have anti-

Fig. 3. Summary of some of the principal factors involved in the regulation of angiogenesis in the corpus luteum and the mechanism by which changes in their activity and interactions may explain the divergent processes. Many of the factors that regulate luteal angiogenesis are derived from the luteal hormone-producing cells and act via receptors on the endothelial cells. The principal angiogenic factor, vascular endothelial growth factor (VEGF), acts via the fms-like tyrosine kinase (Flt-1) and kinase insert domain-containing region (KDR) receptors, while the modulators, Ang-1 and Ang-2, act via Tie-2. Metalloproteinases (MMPs) degrade the extracellular matrix to begin the process that leads to the migration and proliferation of endothelial cells so that all hormone-producing cells are served by a capillary blood vessel. The complexity of the process is illustrated by the heterogeneous nature of expression of factors, such as Ang-2 and insulin-like growth factor binding protein 3 (IGFBP-3), in the endothelial cells, indicating autocrine as well as paracrine regulation. TIMP: tissue inhibitors of MMPs. (b) Luteal 'rescue' is likely to be associated with decreased MMP activity and a tendency towards stabilization of the blood vessels, a phenomenon that usually involves the recruitment of pericytes. A decrease in the synthesis of the angiogenic factors usually follows functional luteolysis (c). An exception may be Ang-2 which, under these conditions, may cause destabilization of the newly formed blood vessels and endothelial cell death. 


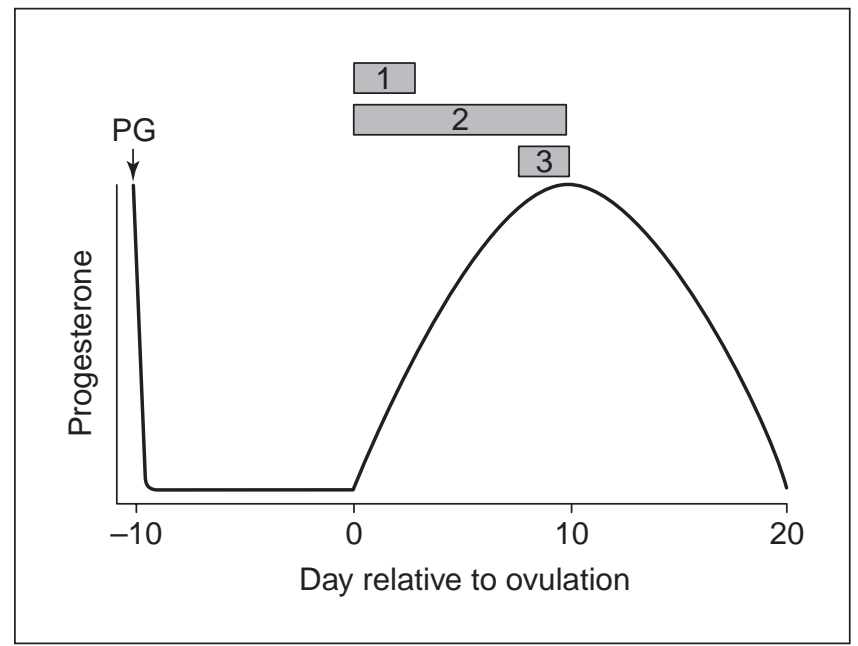

Fig. 5. Schematic showing the different treatment regimens in the marmoset model designed to determine whether inhibition of angiogenic factors or their receptors can prevent angiogenesis ( 1 and 2) or intervene once the process is underway (3).

angiogenic activity; (ii) specific inhibitors of angiogenic factors or their receptors; and (iii) endogenous inhibitors that can be synthesized using recombinant technology (Fraser and Lunn, 2000). Although some first generation compounds suppress ovarian function in mice (Klauber et al., 1997), one of these, TNP-470, did not have a detectable effect on angiogenesis in primates (Fraser et al., 1999a). Because of the large number of compounds in this category, it is important that the consequences of such treatments be assessed, in terms of reproductive function, especially since several of these compounds are now undergoing clinical trials.

Our recent studies have taken advantage of the considerable opportunities afforded by antagonists developed specifically to target individual angiogenic factors. For an understanding of the physiology of angiogenesis, such compounds are ideal, since specific factors may be targeted, their role clarified, and overall controlling pathways unravelled. The role of VEGF in luteal angiogenesis was examined by inhibiting its action with a monoclonal antibody and using three different treatment schedules (Fig. 5). Two of the three treatments involved administration of antibody at or shortly after ovulation, so that VEGF was immunoneutralized during a known period of intense luteal angiogenesis. The first schedule was over a 3 day period, so that the corpus luteum could be examined at a time of normally maximal proliferative activity. The second schedule continued this treatment to the mid-luteal phase, by which time the microvascular tree is established in normal animals, while the third schedule addressed the issue of whether inhibition of VEGF intervenes with ongoing angiogenesis by delaying treatment until days $7-10$ of the luteal phase. The initial short-term treatment reduced the number of proliferating cells markedly (on the basis of incorporation of BrdU into nuclei) (Fig. 4), while the more prolonged treatment resulted in a change in the vascular tree: the larger vessels were still present, but there was a dearth of the numerous small capillaries present in the normal corpus luteum (Fig. 6) (Fraser et al., 2000b). This finding was confirmed by quantitative analysis of endothelial cell area. Treatment with anti-VEGF antibody from day 7 to day 10 also interfered with angiogenesis, with a $70 \%$ reduction in the number of proliferating cells compared with the control corpus luteum at this stage (Dickson et al., 2001). In contrast to the situation observed for treatment with the $\mathrm{GnRH}$ antagonist, the morphological features of the lutein cells in the anti-VEGF antibody-treated animals remained essentially intact; however, the lutein cells appeared to be more closely packed, probably as a direct result of the reduction in the numbers of endothelial cells. In addition, some of the lutein cells exhibited lipid accumulation, while a few cell clusters showed condensation of both nucleus and cytoplasm (Fraser et al., 2000b). Significantly, anti-VEGF treatment is associated with a $60 \%$ reduction in the concentrations of plasma progesterone (Fig. 6) (Fraser et al., 2000b). Overall, these data demonstrate that inhibition of VEGF during early luteal development prevents the intense angiogenesis that normally occurs at this time, leading to a failure of development of the microvascular tree. This failure, in turn, has an adverse effect on the corpus luteum, severely restricting its ability to secrete progesterone. An additional factor contributing to the decrease in progesterone secretion may be a suppression of ovarian microvascular permeability after anti-VEGF treatment (Brasch and Turetschek, 2000), but this possibility remains to be investigated.

\section{Conclusions}

The processes regulating the angiogenic pathway are extremely complex and we recognize that we have presented a simplified overview; however, our objective was to present a working hypothesis in which individual factors could be examined with respect to their potential physiological function. For example, we have illustrated how the role of individual angiogenic factors such as VEGF may be explored using a highly sensitive in vivo model. Because of the marked effect of inhibition of angiogenesis on luteal function, it is predicted that the normal processes of follicular development, ovulation and luteal function could all be profoundly influenced by the manipulation of angiogenesis.

In addition, it is accepted that other factors shown to be associated with the regulation of angiogenesis, which we have been unable to discuss in this short review, may play an essential role in orchestrating the diverse changes in the vasculature of the corpus luteum that occur over a short period. Future studies may reveal shortcomings in the working hypothesis; however, the establishment of the in 


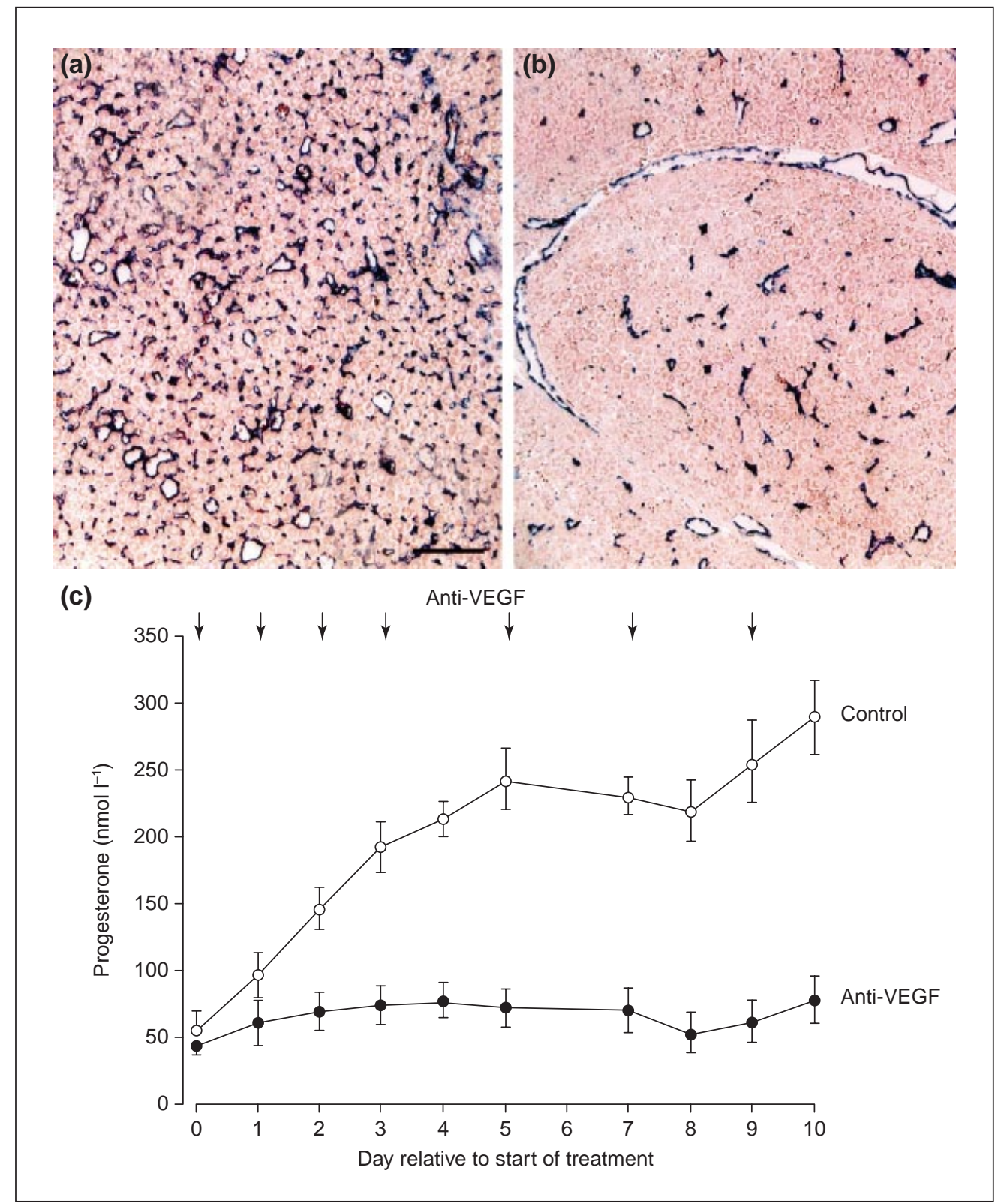

Fig. 6. Photomicrographs of mid-luteal phase marmoset corpora lutea showing localization of factor VIII (dark staining) in the endothelial cells of microvessels and capillaries in a control corpus luteum (a), and reduced staining, particularly for the incidence of capillary endothelial cells, after anti-vascular endothelial growth factor (VEGF) treatment for 10 days (b). Scale bar represents $100 \mu \mathrm{m}$. (c) Plasma progesterone concentrations in control $(\bigcirc)$ and after anti-VEGF treatment for 10 days $(-)$. Treatment started on the day of expected ovulation and is associated with a significant suppression $(P<0.001)$. Values are the mean $\pm \operatorname{SEM}(n=6$ per group). (Adapted from Fraser et al., 2000b.)

vivo model should provide important insights into the physiological role of such putative regulators.

The authors thank their colleagues in the Centre for Reproductive Biology, especially S. E. Dickson, C. Wulff and W. C. Duncan, and collaborators R. Bicknell and G. F. Erickson for their contributions during the course of these investigations. They are also grateful to $\mathrm{H}$. Wilson for technical expertise and E. Pinner for artwork.

\section{References}

Key references are identified by asterisks.

Abylafia O and Sherer DM (2000) Angiogenesis in the ovary American Journal of Obstetrics and Gynecology 182 240-246

Augustin HG (2000) Development of the vascular sytem of the corpus luteum. In Morphogenesis of Endothelium pp 237-254 Ed. W Risau. Harwood Academic Publishers, Reading

Azmi TI and O'Shea JD (1984) Mechanism of deletion of endothelial cells during regression of the corpus luteum Laboratory Investigation $\mathbf{5 1}$ 206-217 
Brasch R and Turetschek K (2000) MRI characterisation of tumours and grading angiogenesis using macromolecular contrast media: status report European Journal of Radiology 34 148-155

Christenson LK and Stouffer RL (1996a) Proliferation of microvascular endothelial cells in the primate corpus luteum during the menstrual cycle and simulated early pregnancy Endocrinology 137 367-374

Christenson LK and Stouffer RL (1996b) Isolation and culture of microvascular endothelial cells from the primate corpus luteum Biology of Reproduction 55 1397-1404

*Dickson SE and Fraser HM (2000) Inhibition of early luteal angiogenesis by gonadotropin-releasing hormone antagonist treatment in the primate Journal of Clinical Endocrinology and Metabolism $852339-2344$

Dickson SE, Bicknell R and Fraser HM (2001) Mid-luteal angiogenesis and function in the primate is dependent on VEGF Journal of Endocrinology 168

Duncan WC (2000) The human corpus luteum: remodelling during luteolysis and maternal recognition of pregnancy Reviews of Reproduction 5 12-17

Eberhard AE, Kahlert S, Goede V, Hemmerlin B, Plate KH and Augustin HG (2000) Heterogeneity of angiogenesis and blood vessel maturation in human tumours: implications for antiangiogenic tumor therapies Cancer Research 60 1388-1393

Ferrara N, Chen H, Davis-Smyth T, Hans-Peter G, Nguyen T-N, Peers D, Chisholm V, Hillan K and Schwall R (1998) Vascular endothelial growth factor is essential for corpus luteum angiogenesis Nature Medicine $\mathbf{4}$ 336-340

Fraser HM and Lunn SF (2000) Angiogenesis and its control in the female reproductive system British Medical Bulletin 56 787-797

Fraser HM, Lunn SF, Kim H and Erickson GF (1998) Insulin-like growth factor binding protein-3 (IGFBP-3) mRNA in the endothelial cells of the primate corpus luteum Human Reproduction 13 2180-2185

Fraser HM, Dickson SE, Morris KD, Erickson GF and Lunn SF (1999a) The effects of the angiogenesis inhibitor TNP-470 on luteal establishment and function in the primate Human Reproduction 14 2054-2060

Fraser HM, Lunn SF, Harrison DJ and Kerr JB (1999b) Luteal regression in the primate: different forms of cell death during natural and gonadotropin-releasing hormone antagonist or prostaglandin analogueinduced luteolysis Biology of Reproduction 61 1468-1479

Fraser HM, Lunn SF, Kim H, Duncan WC, Rodger FE, Illingworth PJ and Erickson GF (2000a) Changes in insulin-like growth factor binding protein-3 mRNA expression in endothelial cells of the human corpus luteum: a possible role in luteal development and rescue Journal of Clinical Endocrinology and Metabolism 85 1672-1677

*Fraser HM, Dickson SE, Lunn SF, Wulff C, Morris KD, Carroll V and Bicknell R (2000b) Suppression of luteal angiogenesis in the primate after neutralization of vascular endothelial growth factor Endocrinology 141 995-1000

Gaytán F, Morales C, García-Pardo L, Reymundo C, Bellido C and Sánchez-Criado JE (1999) A quantitative study of changes in the human corpus luteum microvasulature during the menstrual cycle Biology of Reproduction $60914-919$

Goede V, Schmidt T, Kimmina S, Kozian D and Augustin HG (1998) Analysis of blood vessel maturation processes during cyclic ovarian angiogenesis Laboratory Investigation 78 1385-1394

Hanahan D (1997) Signaling vascular morphogenesis and maintenance Science 277 48-50

Hazzard T, Molskness T, Chaffin C and Stouffer R (1999) Vascular endothelial growth factor (VEGF) and angiopoietin regulation by gonadotrophin and steroids in macaque granulosa cells during the periovulatory interval Molecular Human Reproduction 5 1115-1121

*Klauber N, Rohan RM, Flynn E and D'Amato RJ (1997) Critical components of the female reproductive pathway are suppressed by the angiogenesis inhibitor AGM-1470 Nature Medicine 3 443-446

*Maisonpierre PC, Suri C, Jones PF et al. (1997) Angiopoietin-2, a natural antagonist for Tie2 that disrupts in vivo angiogenesis Science 277 55-60

Modlich U, Kaup FJ and Augustin HG (1996) Cyclic angiogenesis and blood vessel regression in the ovary: blood vessel regression during luteolysis involves endothelial cell detachment and vessel occlusion Laboratory Investigation 74 771-780

Otani N, Sawako M, Yamoto M, Shikone T, Otani H, Nishiyama R, Otani T and Nakano R (1999) The vascular endothelial growth factor/fms-like tyrosine kinase system in human ovary during the menstrual cycle and early pregnancy Journal of Clinical Endocrinology and Metabolism $\mathbf{8 4}$ 3845-3851

Redmer DA and Reynolds LP (1996) Angiogenesis in the ovary Reviews of Reproduction 1 182-192

Reynolds LP, Grazul-Bilska AT and Redmer DA (2000) Angiogenesis in the corpus luteum Endocrine 12 1-9

Rodger FE, Young FM, Fraser HM and Illingworth PJ (1997) Endothelial cell proliferation follows the mid-cycle luteinizing hormone surge but not human chorionic gonadotrophin rescue, in the human corpus luteum Human Reproduction 12 1723-1729

Suzuki T, Sasano H, Takaya R, Fukaya T, Yajima A and Nagura H (1998) Cyclic changes of vasculature and vascular phenotypes in normal human ovaries Human Reproduction 13 953-959

Wiegand SJ, Boland P and Yancopoulos GD (2000) Cooperative roles of the angiopoietins and vascular endothelial growth factor in ovarian angiogenesis. In Ovulation: Evolving Scientific and Clinical Concepts pp 175-186 Ed. EYAdashi. Springer-Verlag, New York

*Wulff C, Wilson H, Largue P, Duncan WC, Armstrong DG and Fraser HM (2000) Angiogenesis in the human corpus luteum: localization and changes in angiopoietins, Tie-2 and vascular endothelial growth factor messenger ribonucleic acid Journal of Clinical Endocrinology and Metabolism 85 4302-4309

Young FM, Rodger FE, Illingworth PJ and Fraser HM (2000) Cell proliferation and vascular morphology in the marmoset corpus luteum Human Reproduction 15 557-566 